\title{
Determining distances using asteroseismic methods
}

\author{
V. Silva Aguirre ${ }^{1,2, \star}$, L. Casagrande ${ }^{3}$, S. Basu ${ }^{4}$, T. L. Campante $^{5,6}$, W. J. Chaplin ${ }^{6,1}$, D. Huber ${ }^{7}$, \\ A. Miglio ${ }^{6}$, A. M. Serenelli ${ }^{8}$, and KASC WG\#1 \\ 1 Stellar Astrophysics Centre, Department of Physics and Astronomy, Aarhus University, Ny Munkegade 120, DK-8000 \\ Aarhus C, Denmark \\ 2 Max Planck Institute for Astrophysics, Karl-Schwarzschild-Str. 1, 85748, Garching bei München, Germany \\ 3 Research School of Astronomy \& Astrophysics, Mount Stromlo Observatory, The Australian National University, ACT \\ 2611, Australia \\ 4 Department of Astronomy, Yale University, P.O. Box 208101, New Haven, CT 06520-8101, USA \\ 5 Centro de Astrofísica and Faculdade de Ciências, Universidade do Porto, Rua das Estrelas, 4150-762 Porto, Portugal \\ ${ }^{6}$ School of Physics and Astronomy, University of Birmingham, Birmingham, B15 2TT, UK \\ 7 NASA Ames Research Center, Moffett Field, CA 94035, USA \\ 8 Instituto de Ciencias del Espacio (CSIC-IEEC), Facultad de Ciències, Campus UAB, 08193 Bellaterra, Spain
}

\section{Received XXXX, accepted XXXX}

Published online XXXX

Key words stars: distances, stars: oscillations, stars: fundamental parameters, techniques: photometric.

\begin{abstract}
Asteroseismology has been extremely successful in determining the properties of stars in different evolutionary stages with a remarkable level of precision. However, to fully exploit its potential, robust methods for estimating stellar parameters are required and independent verification of the results is needed. In this talk, I present a new technique developed to obtain stellar properties by coupling asteroseismic analysis with the InfraRed Flux Method. Using two global seismic observables and multi-band photometry, the technique determines masses, radii, effective temperatures, bolometric fluxes, and thus distances for field stars in a self-consistent manner. Applying our method to a sample of solar-like oscillators in the Kepler field that have accurate Hipparcos parallaxes, we find agreement in our distance determinations to better than $5 \%$. Comparison with measurements of spectroscopic effective temperatures and interferometric radii also validate our results, and show that our technique can be applied to stars evolved beyond the main-sequence phase.
\end{abstract}

(c) 2013 WILEY-VCH Verlag GmbH \& Co. KGaA, Weinheim

\section{Introduction}

The launch of the CoRoT (Baglin et al., 2006) and Kepler missions (Gilliland et al., 2010) produced an authentic revolution in the amount and quality of data on stellar oscillations. From the thousands of light curves obtained by these space missions asteroseismology, the study of pulsations in stars, allows us to obtain accurate stellar parameters using two global oscillation observables.

The power spectrum of solar-like oscillators is modulated in frequency by a Gaussian-like envelope, from where the frequency of maximum power $\nu_{\max }$ can be readily extracted. The near-regular pattern of high overtones presents a dominant frequency spacing called the large frequency separation, $\Delta \nu$. Applying scaling relations from solar values, these two asteroseismic observables may be used to estimate stellar properties of large numbers of solar-like oscillators (e.g., Basu et al., 2010; Stello et al., 2008).

In this talk I present a new method to derive stellar parameters, including distances, in a self-consistent manner by combining seismic determinations to the Casagrande et al. (2010) implementation of the InfraRed Flux Method

\footnotetext{
^ Corresponding author: victor@phys.au.dk
}

(IRFM). Our results are compared with Hipparcos parallaxes, high-resolution spectroscopic temperature determinations, and interferometric measurements of angular diameters. All the relevant details and initial applications of this technique can be found in Silva Aguirre et al. (2011, 2012).

\section{Obtaining asteroseismic stellar parameters}

The large frequency separation $\Delta \nu$ scales as the square root of the mean density (e.g., Ulrich, 1986), while $\nu_{\max }$ is related to the acoustic cutoff frequency of the atmosphere (e.g., Brown et al., 1991; Kjeldsen \& Bedding, 1995). Based on the accurately known solar parameters, two scaling relations can be written for these quantities (e.g., Hekker et al., 2009)

$$
\begin{aligned}
\frac{M}{M_{\odot}} \simeq\left(\frac{\nu_{\max }}{\nu_{\max , \odot}}\right)^{3}\left(\frac{\Delta \nu}{\Delta \nu_{\odot}}\right)^{-4}\left(\frac{T_{\mathrm{eff}}}{T_{\mathrm{eff}, \odot}}\right)^{3 / 2}, \\
\frac{R}{R_{\odot}} \simeq\left(\frac{\nu_{\max }}{\nu_{\max , \odot}}\right)\left(\frac{\Delta \nu}{\Delta \nu_{\odot}}\right)^{-2}\left(\frac{T_{\mathrm{eff}}}{T_{\mathrm{eff}, \odot}}\right)^{1 / 2},
\end{aligned}
$$

where $T_{\text {eff }, \odot}=5777 \mathrm{~K}, \Delta \nu_{\odot}=135.1 \pm 0.1 \mu \mathrm{Hz}$ and $\nu_{\max , \odot}=3090 \pm 30 \mu \mathrm{Hz}$ are the values observed in the Sun 
(Huber et al., 2011). If we can determine the $T_{\text {eff }}$ value, for instance via the IRFM, these relations give a determination of stellar mass and radius for each star that is independent of evolutionary models (see, e.g., Miglio et al., 2009).

The basic idea behind the IRFM is to recover for each star its bolometric $\left(\mathcal{F}_{\mathrm{Bol}}\right)$ and infrared monochromatic flux $\left(\mathcal{F}_{\lambda_{\mathrm{IR}}}\right)$, both measured at the top of Earth's atmosphere. One then compares their ratio to that obtained from the same quantities defined on a surface element of the star, i.e. the bolometric flux $\sigma T_{\text {eff }}^{4}$ and the theoretical surface infrared monochromatic flux. Once $\mathcal{F}_{\mathrm{Bol}}$ (Earth) and $T_{\text {eff }}$ are both known, the limb-darkened angular diameter, $\theta$, is trivially obtained. In the adopted implementation, the bolometric flux was recovered using multi-band photometry, and the flux outside of these bands (i.e., the bolometric correction) was estimated using a theoretical model flux from the Castelli \& Kurucz (2004) grid. Thus the method relies on the input $[\mathrm{Fe} / \mathrm{H}]$ and $\log g$, and an iterative process in $T_{\text {eff }}$ to match the observed $\mathcal{F}_{\mathrm{Bol}}$.

As an initial sample to test the accuracy of our technique, we selected the stars that have accurate Hipparcos parallaxes (van Leeuwen, 2007), which accounts for only 21 targets out of the more than 500 main-sequence and subgiant stars in which Kepler detected oscillations in its shortcadence mode (Chaplin et al., 2011). The asteroseismic observables $\Delta \nu$ and $\nu_{\max }$ were obtained from the power spectra using the pipeline described by Huber et al. (2009). To recover the bolometric flux, we used multi-band $B_{T} V_{T}$ and $J H K_{S}$ photometry from the Tycho2 (Høg et al., 2000) and Two Micron All Sky Survey (2MASS; Skrutskie et al., 2006) catalogs, respectively. The infrared monochromatic flux was derived from 2MASS $J H K_{S}$ magnitudes only.

To apply the IRFM the metallicity of the targets must be given as an input. We have chosen the chemical composition of the targets in the following order of preference, according to availability: the latest revision of the GenevaCopenhagen Survey (GCS, Casagrande et al., 2011), spectroscopic determinations from Bruntt et al. (2012), or the value given in the Kepler Input Catalogue (KIC; Brown et al., 2011) increased by 0.18 dex. The latter is the offset found between GCS and the KIC for the 11 stars common in our sample, and is similar to the +0.21 dex offset found by Bruntt et al. (2012). Reddening must also be specified, and our calculations were made using the distance dependent three-dimensional Galactic extinction model from Drimmel et al. (2003). The values were obtained after an iteration in distance as described by Miglio et al. (2012b).

From the previous paragraphs it is clear that the asteroseismic method provides a mass and radius based on an input $T_{\text {eff }}$ value, while the IRFM gives $T_{\text {eff }}$ and the bolometric flux at a given input $\log g$ and $[\mathrm{Fe} / \mathrm{H}]$. In order to determine a unique set of stellar parameters for each star, we iterated the two methods in a consistent way. We started by calculating sets of IRFM effective temperatures for each star at fixed $\log g=2.0-5.0$ in steps of 0.5 dex; this translates into $T_{\text {eff }}$ changes of less than $1 \%$ for each $\log g$ step. Using $\log g$ determinations from the KIC as an initial guess, we interpolated in gravity and computed $T_{\text {eff }}$ from the IRFM results. This $T_{\text {eff }}$ value, together with $\nu_{\max }$ and $\Delta \nu$, was fed to the scaling relations to obtain a mass, radius, and thus $\log g$. Interpolating again in gravity gave an updated value of $T_{\text {eff }}$, and the procedure was repeated until convergence in $\log g$ and $T_{\text {eff }}$ was reached.

The $1 \sigma$ uncertainties of the parameters were obtained during the iterations. We took into account both the uncertainties in the seismic observables, as well as variations in the $T_{\text {eff }}$ determinations arising from different photometric filters and $\log g$ determinations. The results are affected by the assumed value of extinction, and are very mildly dependent on the metallicity considered. To account for possible errors in reddening and composition, we have also computed sets of IRFM effective temperatures at $\log g=3.5$, one increasing $E(B-V)$ by +0.01 (the decreasing case is basically symmetric), and another one changing the metallicity by \pm 0.1 dex. Moreover, a Monte-Carlo simulation was run to estimate the uncertainties in $T_{\text {eff }}$ from random photometric errors. Finally, an extra $20 \mathrm{~K}$ were added to the error budget to account for the uncertainty in the zero-point of the temperature scale (see Casagrande et al., 2010).

\section{Results}

Using the asteroseismic radius and the limb-darkened angular diameter $\theta$, it is straightforward to estimate the distance:

$$
d_{\text {seis }}=C \frac{2 R}{\theta},
$$

where $C$ is the conversion factor to parsecs. In this manner, we determined asteroseismic distances for our sample targets. Fig. 1 shows the comparison between our asteroseismic distances and those obtained from Hipparcos parallax measurements. The agreement is excellent, particularly for the close-by targets, boosting our confidence on the asteroseismic parameters and the robustness of our technique. The weighted mean difference (Hipp. - Seis.) is $2.3 \% \pm 1.8 \%$.

For the method to be self-consistent, we must make sure that our angular diameter and $T_{\text {eff }}$ determinations are also robust. Bruntt et al. (2012) made a spectroscopic analysis of all but one of our targets, obtaining effective temperatures via the excitation balance of Fe I lines at a fixed $\log g$ as determined by asteroseismology. In Fig. 2 2 we compare their $T_{\text {eff }}$ values with ours and find excellent agreement. Individual fractional differences are below $2 \%$, while the weighted mean difference (Spec. - Seis.) is $-0.8 \% \pm 0.4 \%$. This level of agreement is particularly impressive considering that the uncertainties quoted by Bruntt et al. (2012) are of $70 \mathrm{~K}$ for all the targets.

We can verify the results of our technique by comparing our derived angular diameters with results from interferometry. In a recent observation run carried out at the PAVO/CHARA long-baseline interferometer, Huber et al. (2012) analyzed a total of ten asteroseismic targets, four of which are contained in our sample. We also applied our 


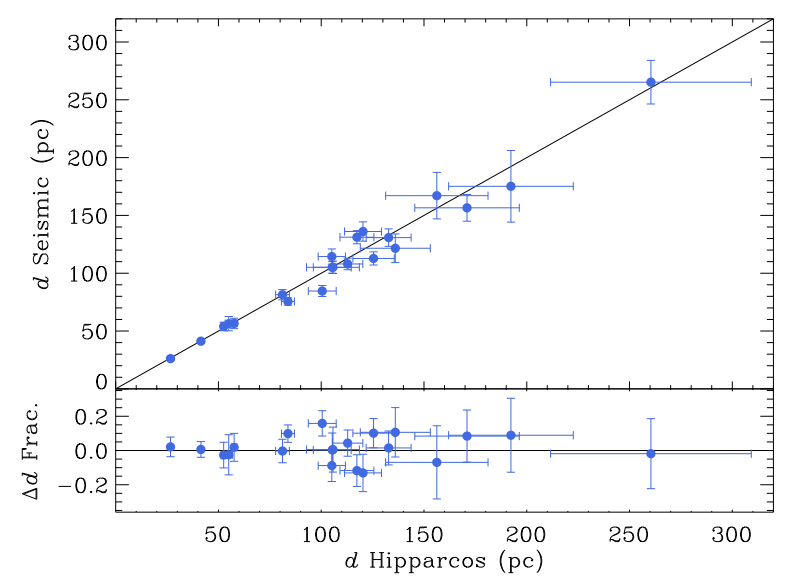

Fig. 1 Upper panel: comparison of Hipparcos distances with those obtained via the seismic method. Lower panels: fractional difference (Hipp. - Seis.) between both determinations. Solid lines show the one-to-one correspondence.

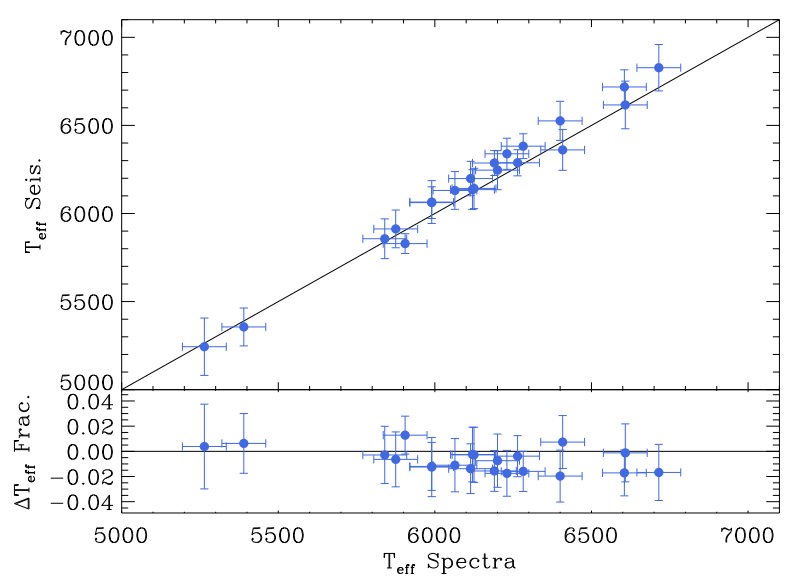

Fig. 2 Upper panel: comparison of effective temperatures using the asteroseismic method with those obtained from spectroscopy by Bruntt et al. (2012). Lower panel: fractional difference (Spec. - Seis.) between both determinations. Solid lines shows the one-to-one correspondence.

technique to the remaining six stars from the Huber et al. (2012) analysis, that correspond to more evolved red giants targets. In Fig. 3 we show the comparison between the angular diameters derived from interferometry and our technique, which agree remarkably well for main-sequence and red giant evolutionary phases (residual mean of $-2 \% \pm 2 \%$ ). More details can be found in Huber et al. (2012).

After verifying our technique via parallax, spectroscopic, and interferometric measurements, we applied our procedure to the full short-cadence Kepler sample and derived consistent parameters, including distances, for all these stars. The 565 targets considered are predominantly

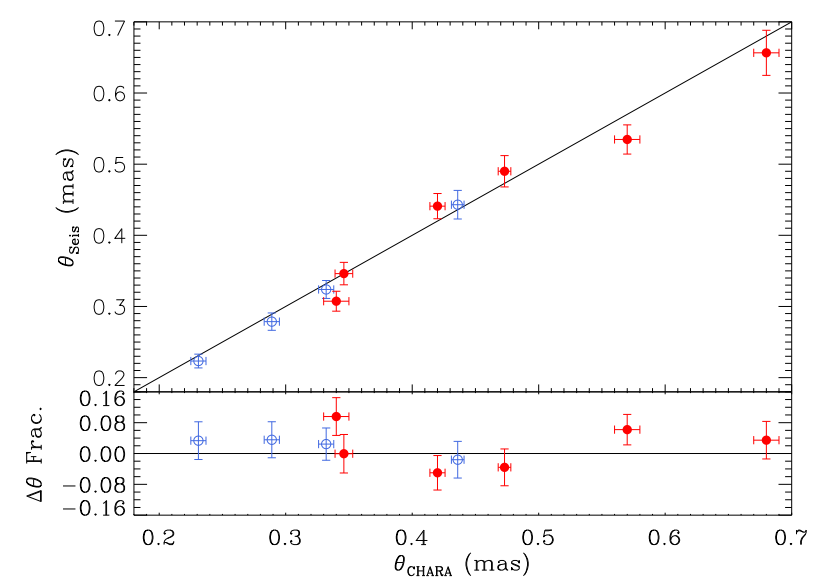

Fig. 3 Upper panel: angular diameters comparison of those derived by our technique to the ones obtained from spectroscopy by Huber et al. (2012). Lower panel: fractional difference (CHAR. - Seis.) between both determinations. Solid lines shows the one-to-one correspondence. Light open circles show the targets analyzed in this paper, while the dark filled circles depict those evolved red giants from the Huber et al. (2012) sample. See text for details.

main-sequence and subgiant stars, with a handful of red giants also present in the sample. Nevertheless, as shown in Fig. 3 our results are clearly applicable to stars evolved beyond the turnoff. All the targets have available Tycho2 photometry, and we have used metallicites from the KIC increased by 0.18 dex. To account for the uncertainties in composition, we computed IRFM sets of $T_{\text {eff }}$ varying the metallicity by \pm 0.3 dex. In Fig. 4 we show a histogram with the obtained distance distribution. The results shows that we can use Kepler data to probe populations of main-sequence and subgiant field stars as far as $1 \mathrm{kpc}$ from the Sun.

\section{Conclusions}

Determining accurate stellar parameters is crucially important for detailed studies of individual stars, as well as for characterizing stellar populations in the Milky Way. The asteroseismic revolution produced by the CoRoT and Kepler missions requires robust techniques to exploit fully the potential of the data and provide the community with the necessary information for ensemble analysis.

Using oscillation data and multi-band photometry, I have presented in this talk a new method to derive stellar parameters combining the IRFM with asteroseismic analysis. The novelty of our approach is that it allows us to obtain radius, mass, $T_{\text {eff }}$, and bolometric flux for individual targets in a self-consistent manner. This naturally results in direct determinations of angular diameters and distances without resorting to parallax information, further enhancing the capabilities of our technique. 


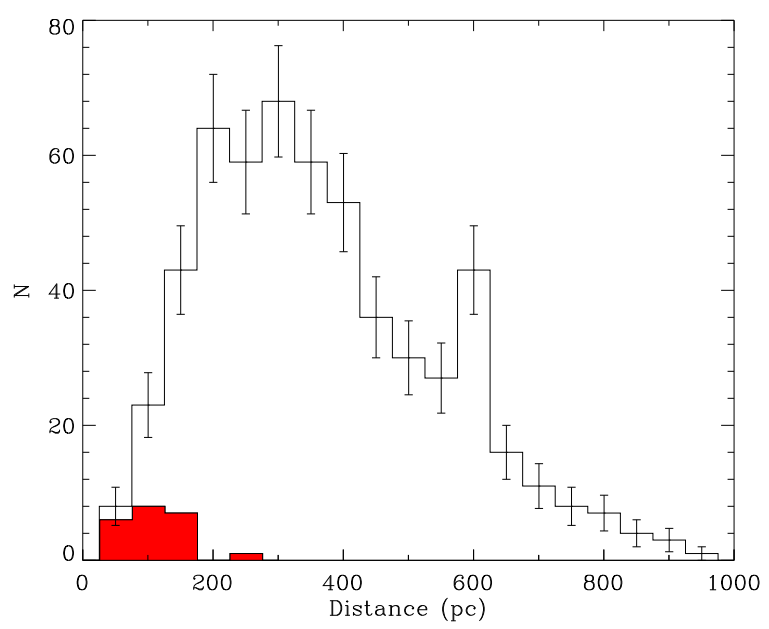

Fig. 4 Distance distribution derived using our technique for the complete Kepler short-cadence sample (unshaded region). The shaded region shows the results for the 21sample stars as a comparison.

Comparison of our distance results with those from Hipparcos parallaxes shows an overall agreement better than $3 \%$. Furthermore, the obtained $T_{\text {eff }}$ values show a mean difference below $1 \%$ when compared to results from highresolution spectroscopy. We have also compared our calculated angular diameters with those measured by longbaseline interferometry and found agreement better than $5 \%$. This provides verification of our radii, $T_{\text {eff }}$ and bolometric fluxes to an excellent level of accuracy, and shows that the results for red giant stars are also accurate.

Studies of the stellar populations in the CoRoT and Kepler fields can greatly benefit from accurate masses, radii, $T_{\text {eff }}$, and distances (Miglio, 2012a; Miglio et al., 2012b). Combining this information with stellar evolutionary models and metallicity measurements can lead to an age-metallicity relation, opening the possibility of testing models of Galactic Chemical Evolution in stars outside the solar neighborhood (e.g., Chiappini et al., 1997; Schönrich \& Binney, 2009). Applying our method to the complete short-cadence Kepler sample reveals that we can probe stars as far as $1 \mathrm{kpc}$ from our Sun, making this set of main-sequence and subgiant stars extremely interesting for population studies. Although much greater distances can be probed by analyzing oscillations in giants, the ages of these stars are mostly determined by their main-sequence lifetime (e.g., Salaris et al., 2002). Thus, the short-cadence sample is of key importance for helping to calibrate absolute mass-age relationships of red giants and correctly characterize their populations.

Acknowledgements. Funding for the Kepler Discovery mission is provided by NASA's Science Mission Directorate. The authors wish to thank the entire Kepler team, without whom these results would not be possible. We also thank all funding councils and agencies that have supported the activities of KASC Working Group 1. We are also grateful for support from the International
Space Science Institute (ISSI). V.S.A. received financial support from the Excellence cluster "Origin and Structure of the Universe" (Garching)

\section{References}

Baglin, A., Auvergne, M., Barge, P., et al. 2006, in ESA SP 1306, 33

Basu, S., Chaplin, W. J., \& Elsworth, Y. 2010, ApJ , 710, 1596

Brown, T. M., Gilliland, R. L, Noyes, R. W., \& Ramsey, L. W. 1991, ApJ , 368, 599

Brown, T. M., Latham, D. W., Everett, M. E, \& Esquerdo, G. A. 2011, AJ , 142, 112

Bruntt, H., Basu, S., Smalley, B., et al. 2012, MNRAS , 423, 122

Casagrande, L., Ramírez, I., Meléndez, J., et al. 2010, A\&A, 512, 54

Casagrande, L., Schönrich, R., Asplund, M., et al. 2011, A\&A, 530, A138

Castelli, F., \& Kurucz, R. L. 2004, arXiv:astro-ph/0405087

Chaplin, W. J., Kjeldsen, H., Christensen-Dalsgaard, J., et al. 2011, Science, 332, 213

Chiappini, C., Matteucci, F., \& Gratton, R. 1997, ApJ , 477, 465

Drimmel, R., Cabrera-Lavers, A., \& López-Corredoira, M. 2003, A\&A, 409, 205

Gilliland, R. L., Brown, T. M., Christensen-Dalsgaard, J., et al. 2010, PASP , 122, 131

Hekker, S., Kallinger, T., Baudin, F., et al. 2009, A\&A, 506, 465

Høg, E., Fabricius, C., Makarov, V. V., et al. 2000, A\&A, 355, L27

Huber, D., Stello, D., Bedding, T. R., et al. 2009, Comm. Aster., 160,74

Huber, D., Bedding, T. R., Stello, D. et al. 2011, ApJ , 743, 143

Huber, D., Ireland, M. J., Bedding, T. R., et al. 2012, ApJ , in press (arXiv:1210.0012)

Kjeldsen, H. \& Bedding, T. R. 1995, A\&A, 293, 87

Miglio, A., Montalban, J., Baudin, F. et al. 2009, A\&A, 503, L21

Miglio, A. 2012a, in Red Giants as Probes of the Structure and Evolution of the Milky Way, Astrophysics and Space Science Proceedings, ed. A. Miglio, J. Montalban, \& A. Noels (Springer-Verlag Berlin Heidelberg),p.11

Miglio, A., Chiappini, C., Morel, T. et al. 2012b, MNRAS , submitted

Salaris, M., Cassisi, S., \& Weiss, A. 2002, PASP , 114, 375

Schönrich, R., \& Binney, J. 2009, MNRAS , 396, 203

Silva Aguirre, V., Chaplin, W. J., Ballot, et al. 2011, ApJ , 740, L2

Silva Aguirre, V., Casagrande, L., Basu, S., et al. 2012, ApJ , 757, 99

Skrutskie, M. ,F., Cutri, R. M., Stiening, R., et al. 2006, AJ , 131, 1163

Stello, D., Bruntt, H., Preston, H., \& Buzasi, D. 2008, ApJ , 674, L53

Ulrich, R. K. 1986, ApJ , 306, L37

van Leeuwen, F. 2007, A\&A, 474, 653 